\title{
GRAIN BOUNDARY DESIGN FOR ADVANCED MATERIALS ON THE BASIS OF THE RELATIONSHIP BETWEEN TEXTURE AND GRAIN BOUNDARY CHARACTER DISTRIBUTION (GBCD)
}

\author{
TADAO WATANABE \\ Department of Materials Science, Faculty of Engineering, Tohoku University, \\ Sendai, Japan
}

\begin{abstract}
On the basis of recent knowledge of structure-dependent boundary properties, the design and control of boundary-related bulk properties and performance of advanced polycrystalline materials are discussed. The grain boundary character distribution (GBCD) which has been recently introduced as a new microstructural factor is shown to be a powerful tool for designing and controlling the bulk properties in polycrystalline materials. The relationship between texture and GBCD has been discussed in connection with other microstructural factors associated with grain boundaries. It has been shown that the relationship between texture and GBCD can provide an important clue to the grain boundary design and control for polycrystalline materials with desirable properties and performance. The present paper shows recent successful achievement of toughening of brittle materials by controlling texture and GBCD. The potential and prospective of the grain boundary design and control for functional materials are also discussed.
\end{abstract}

KEY WORDS Grain boundary character, iron-silicon, coincidence boundaries, grain boundary design.

\section{INTRODUCTION}

In the last two decades the importance of grain and interphase boundaries which may have invaluable potential for materials design and development has been drawing an increasing interest and involvement of many materials scientists and engineers in the research area of Interface Science and Engineering. Grain boundaries (hereafter including interphase boundaries) are important microstructural elements in all kinds of polycrystalline materials whether being structural or functional; metallic materials, semiconductors, electronic and superconducting ceramics and even polymers. It has been well established that grain boundaries play important roles as preferential sites for metallurgical phenomena occurring in the materials. Accordingly the bulk properties of polycrystalline materials can be affected by the presence of grain boundaries and differ from those of single crystal. Recent experimental and theoretical studies of the structure and properties of grain boundaries have revealed that the properties of individual grain boundaries depend strongly on the type and structure of the boundary (Gleiter and Chalmers, 1972, Chadwick and Smith, 1976, Rühle et al., 1985). The influence of grain boundaries on the bulk properties and performance are simply classified into two categories; beneficial and detrimental. From the 
view point of materials design and development it is reasonable to consider that grain boundaries should be utilized to exert their beneficial influence as much as possible, leaving their harmful effects suppressed in order to endow polycrystalline materials with desirable high performance or even a new function. This is the basic idea of the grain boundary design and control for advanced materials proposed by the present author (Watanabe, 1984, 1986, 1988a). For this purpose we need scientific knowledge of structure-dependent properties of grain boundaries and engineering knowledge of the way how to design and control grain boundaries in polycrystals in order to produce desirable properties and performance. The present author proposed a new microstructural factor associated with grain boundaries, that is "the grain boundary character distribution (GBCD)" and systematic investigation into GBCD has been being made (Watanabe 1984, $1988 \mathrm{~b})$. In particular, the recent finding of the close relationship between GBCD and texture (Randle et al., 1988; Watanabe et al., 1989a, 1989b, Watanabe 1990) appears to be able to provide us useful and important information for the grain boundary design and control for advanced materials. The present paper discusses firstly the importance of grain boundary structural effect and GBCD as sources of many boundary-related properties, secondly some factors affecting GBCD. The relationship between GBCD and texture will be discussed in some detail, referring to recent experimental and theoretical studies. Finally we look at recent achievement and future prospective of the grain boundary design and control for several kinds of advanced structural and functional materials.

\section{THE IMPORTANCE OF GRAIN BOUNDARY STRUCTURAL EFFECT TO GRAIN BOUNDARY DESIGN}

In recent years much experimental and theoretical work concerning the effects of boundary type and structure upon boundary properties has been extensively performed by using bicrystals containing well characterized grain boundaries (Gleiter and Chalmers, 1972, Chadwick and Smith, 1976, Balluffi, 1980, Rühle et al., 1985, Raj and Sass, 1988, Yoo et al., 1988). It has been well established that there exists a significant difference in boundary properties between low-energy special boundaries and high-energy random boundaries. Low-energy boundaries are known to have high resistance to diffusion (Biscondi, 1984), segregation (Watanabe et al., 1980), corrosion (Froment, 1975, Yamashita et al., 1991), diffusion-induced-migration (King, 1987), sliding (Lagarde and Biscondi, 1974, Watanabe, 1983b) and fracture (Kurishita and Yoshinaga, 1989, Watanabe, 1984). On the contrary high-energy random boundaries are preferential sites for the metallurgical phenomena. Here it should be mentioned that boundary migration appears to more complicatedly depend on the boundary type and structure, in connection with its driving force under static or dynamic condition (Fukutomi and Horiuchi, 1981, Raman et al., 1989), and the effect of segregation (Aust, 1981). However until recently structural effects of grain boundaries were not seriously taken into account in discussing the properties and performance of polycrystalline materials although important roles of grain boundaries had been well recognized and discussed qualitatively. This is probably because no powerful technique such as SEM-ECP or SEM-EBSP for orientation determination was 
not developed until recently. Accordingly quantitative and statistical analysis of GBCD in polycrystals was practically difficult. Furthermore we did not have any means by which the collective effect of individual grain boundaries can be related to the bulk properties of a polycrystal, from boundary structure point of view. Now we can use the grain boundary character distribution (GBCD) which describes the type and frequency of grain boundaries in a polycrystal. The following section briefly discusses presently available experimental technique for the characterization of grain boundary, the description of GBCD, and some factors affecting GBCD.

\section{GRAIN BOUNDARY CHARACTER DISTRIBUTION (GBCD) CONTROLLING BULK PROPERTIES}

\subsection{The Characterization of Grain Boundaries}

Grain boundaries can be characterized crystallographically and geometrically by the relative orientation relationship between two grains, and classified by the minimum rotation angle (disorientation, $\theta)$ into low-angle boundaries $\left(\theta \leqq 15^{\circ}\right)$ and high-angle boundaries $\left(\theta>15^{\circ}\right)$. Moreover depending on special relative orientation relationship, particularly defined by the coincidence site lattice (CSL) theory, we can also classify grain boundaries into (i) coincidence boundaries with specific $\Sigma$ value which is the reciprocal density of coincidence lattice sites and a measure of the degree of structural order of grain boundary, and (ii) noncoincidence boundaries, so called general random boundaries (Gleiter and Chalmers, 1972). Accordingly for simplicity we usually classify observed grain boundaries into the following three groups; low-angle boundary, coincidence boundary and high-angle random boundary. We take the maximum $\Sigma$ value of 29 for coincidence boundaries which can show some special property from our own experience and the literature on the observations of structure-dependent boundary properties in bicrystals. We may consider both low-angle boundaries and low$\Sigma$ conicidence boundaries as being of low-energy and random boundary as of high energy, on the basis of the observation that low $\Sigma$ coincidence boundaries tend to have low boundary energies (Otsuki and Mizuno, 1986, Dechamps, 1991, Schmelzle et al., 1991) although there still exists the controversy to this (Sutton and Balluffi, 1987) mainly because of the deficiency of direct experimental data on grain boundary energy. The advent of SEM-electron channelling pattern (ECP) technique and other modern ones (Randle et al., 1988) for microscale orientation analysis has enabled us to do grain boundary characterization for many boundaries in polycrystalline materials without much difficulty. Therefore statistical studies of grain boundary character distribution have recently become possible. Figure 1(a) and 1(b) show respectively SEM and SEM-ECP micrographs taken at a grain boundary in a rapidly solidified and annealed ribbon specimen of Fe-6.5 mass \% Si alloy. From the displacement of $\{001\}$ pole in the direction normal to the boundary due to the rotation along $\langle 100\rangle$ axis by about $8^{\circ}$ in the SEM-ECP micrograph, the boundary is characterized as $8^{\circ}\langle 100\rangle$ tilt boundary. Figure 1(c) indicates a ECP micrograph taken from an almost $\langle 100\rangle$ twist boundary in the same material. In this case the $\{001\}$ poles of the two grains are relatively rotated by about $6^{\circ}$ in the direction parallel to the boundary. Normally grain boundary characterization is made by determining the relative orientation 


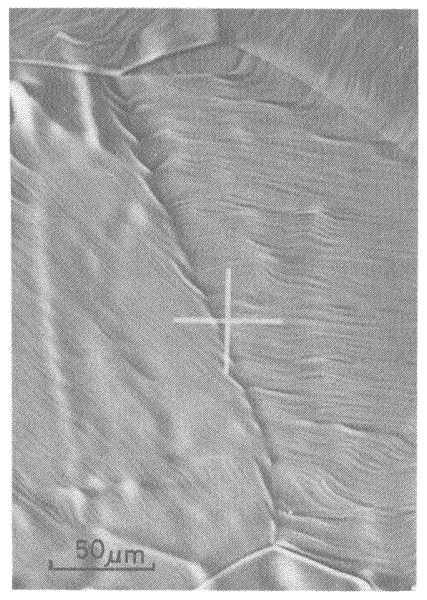

SEM

(a)

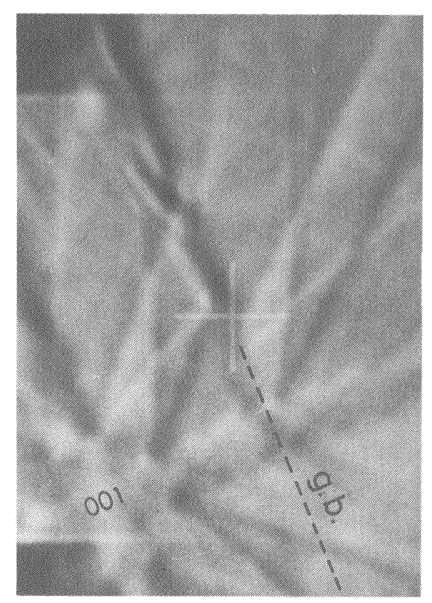

ECP

(b)

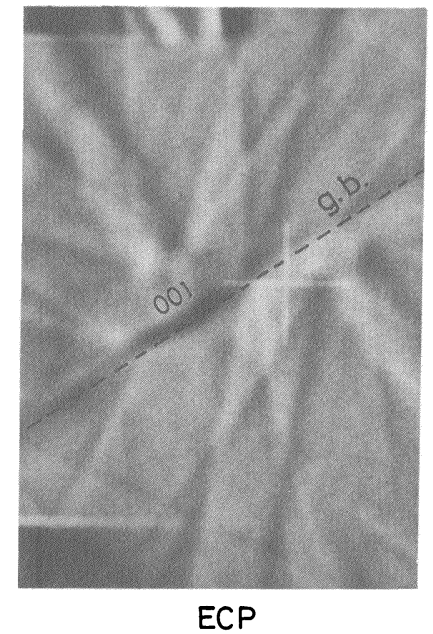

(c)

Figure 1 SEM-ECP micrographs taken at a tilt boundary and a twist boundary in $\mathrm{Fe}-6.5$ mass \% $\mathrm{Si}$ ribbon rapidly solidified and annealed at $1363 \mathrm{~K}$ for $3.6 \mathrm{ks}$. (a) SEM and (b) ECP micrographs taken from a tilt boundary and (c) ECP micrograph from a twist boundary.

of neighbouring grains from their SEM-ECP micrographs by hand or recently by using a digitizer connected to a computer for semi-automatic determination.

Figure 2 is an example of a map of the grain boundary character distribution (GBCD) determined for a molybdenum polycrystal produced by thermomechanical processing from single crystal. Low-angle boundaries are indicated by dotted lines, coincidence boundaries and random boundaries are denoted by $\Sigma$ and $R$, respectively. Large grown grains tend to have more random boundaries while small grains more low-angle boundaries. This suggests that grain growth is likely controlled by the GBCD and the prevailing microstructure in polycrystals. The present author has quite recently discussed the importance of GBCD to grain growth in detail (Watanabe, 1992). In the last decade experimental work on GBCD has been done by increasing number of researchers for metallic, ceramic, semiconductor and superconducting materials, as discussed later in depth.

\subsection{Factors Affecting $G B C D$}

It is interesting to study possible factors affecting GBCD in real polycrystalline materials. The present author and coworkers have made a systematic investigation into this subject and the results have been reported (Watanabe, 1984, 1988a, 1988b). Firstly it was found that there is a close relationship between GBCD and grain size in polycrystalline materials. As shown in Figure 3, the frequency of low $\Sigma$ coincidence boundaries (including low angle or $\Sigma 1$ coincidence boundary) increases with decreasing grain size. The fine-grained tungsten polycrystals with grain size smaller than $10 \mu \mathrm{m}$ can contain more than $30 \%$ low $\Sigma$ coincidence boundaries. Moreover aluminium polycrystals with grain size smaller than $30 \mu \mathrm{m}$ contain more than $50 \%$ low $\Sigma$ coincidence boundaries. The level of 


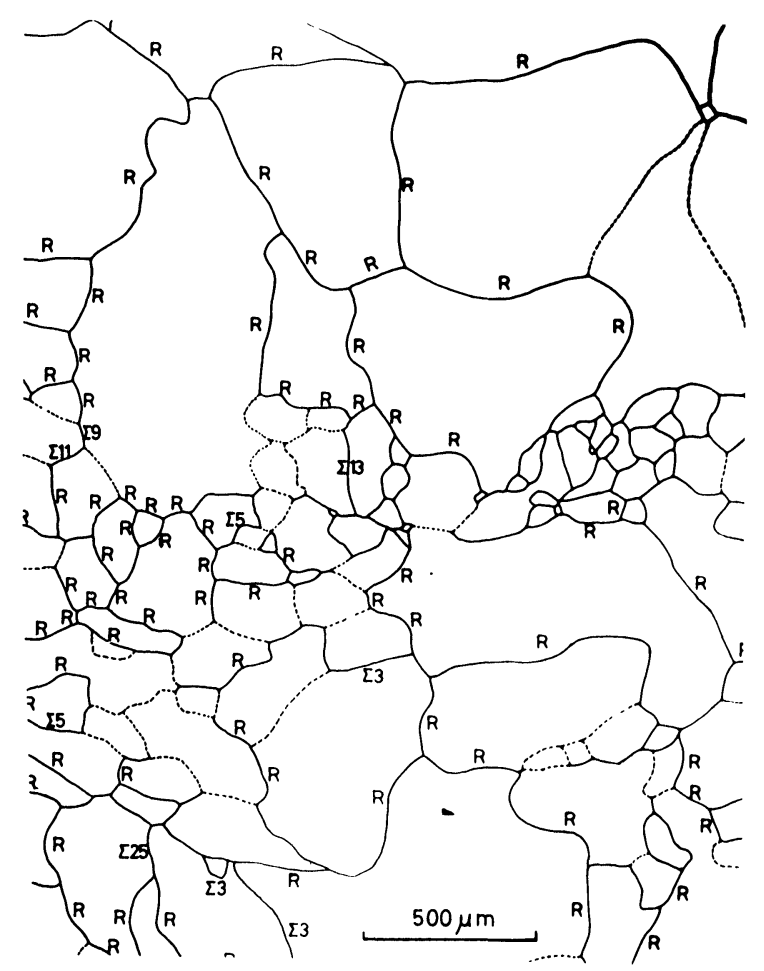

Figure 2 Grain boundary character distribution (GBCD) in a molybdenum polycrystal recrystallized from the single crystal with (110) initial orientation. (80\% deformed under compression and annealed at $1873 \mathrm{~K}$ for $7.2 \mathrm{ks}$ ).

the frequency of low $\Sigma$ coincidence boundaries at a given grain size appears to depend on the material and probably processing method of polycrystals. Those polycrystals which have fine grain size and a high frequency of low energy boundaries are expected to have boundary-related properties different from those of coarse-grained polycrystals. For instance, frequently observed high ductility of fine grained polycrystals is very likely ascribed to the presence of a high frequency of low energy boundaries resistant to fracture. However we must be careful enough not to misunderstand and bear in mind that the relationship between grain size and GBCD has been found to depend on processing and even an inverse relationship exists, as observed between polycrystals produced by thermomechanical processing and by rapid solidification and subsequent annealing (Watanabe et al., 1989a, 1989b). Fine-grained as-rapidly solidfied polycrystal of iron silicon alloy was found to have more random boundaries, as in the case of fine-grained sintered polycrystals. It has also been found that GBCD depends on the crystal structure; in FCC polycrystals $\Sigma 3$-related boundaries where the relative orientations are close to CSL orientations corresponding to $\Sigma 9,27,81$, $\left(\Sigma 3^{n}\right)$ can occur preferentially by multiple twinning (Lim and Raj, 1984). So there exists the difference in GBCD for polycrystals with FCC of low stacking fault energy and BCC of high stacking fault energy (Watanabe et al., 1989b). Another 


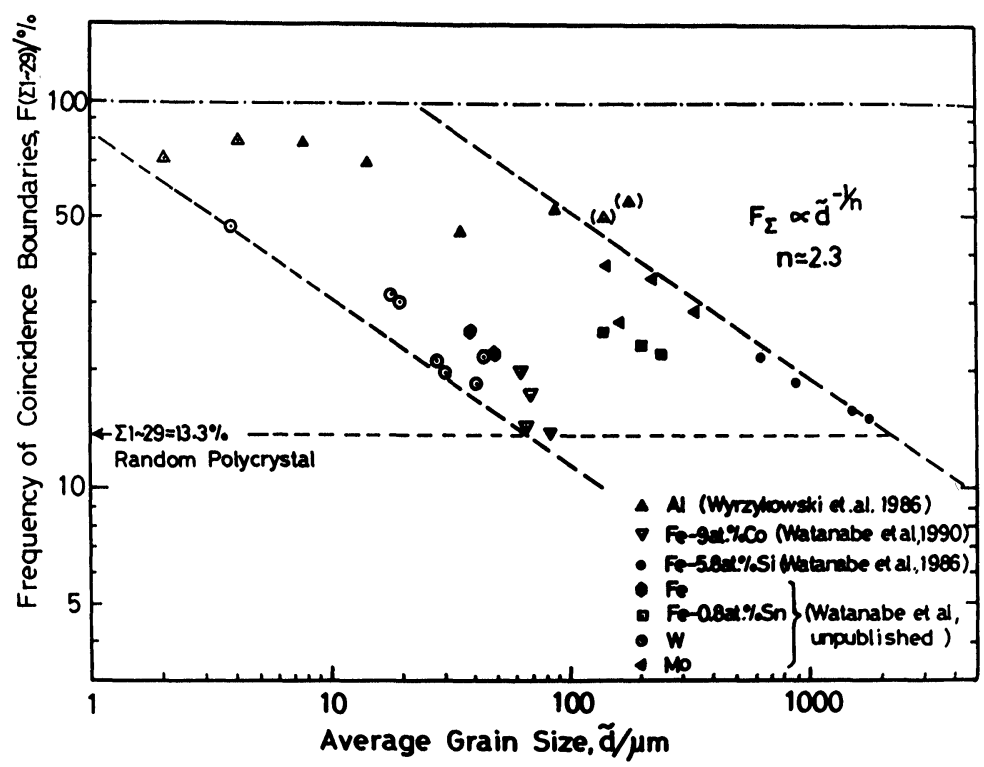

Figure 3 The frequency of coincidence boundaries as a function of the average grain size for recrystallized metallic materials.

interesting factor is microalloying effect. As reported earlier and reconfirmed recently by Aust and coworkers for aluminium and nickel alloys (Belluz and Aust, 1975, Aust and Palumbo, 1989), the addition of very minute (a few tens ppm order) elements can drastically change GBCD in metal polycrystals which are produced by thermomechanical processing. This may be associated with the effect of grain boundary segregation of alloying elements on grain boundary migration occurring during recrystallization involved in thermomechanical processing. Such microalloying effect on GBCD is likely the major origin of microalloying effects on various metallurgical phenomena in many alloys reported so far (Honeycombe, 1983).

\section{RELATIONSHIP BETWEEN TEXTURE AND GBCD}

Until recently the effects of grain boundaries on texture evolution and the properties of textured polycrystalline materials have not been fully understood in connection to the type and structure of grain boundary, and particularly to GBCD although several workers have studied this important subject (Howell et al., 1978, Abbruzzese and Lücke, 1986). It has been reported that low angle boundaries and coincidence boundaries with specific $\Sigma$ play important roles in the evolution of the Goss texture in iron-3\% silicon alloy and iron-nickel alloy (Harase et al., 1988, Shimizu and Harase, 1989). Moreover the plane-matching type boundaries appears to be involved in texture evolution in tungsten (Howell et al., 1978) and iron-silicon alloy (Watanabe, 1983a) with BCC structure. The presence of such low energy special boundaries seems essential for abnormal 
grain growth and subsequent texture evolution (Watanabe, 1992). Quite recently several workers have made systematic investigations of texture and GBCD. It has been found that GBCD is closely related to the type and sharpness of texture in polycrystals (Randle and Brown, 1989, Watanabe et al., 1989a, 1989b, Watanabe, 1990). The frequency of low energy boundaries like low $\Sigma$ coincidence boundaries which occur preferentially in textured polycrystals, was found to be quantitatively predictable by the inverse cubic root $\Sigma$ empirical law. Thus in last several years the relationship between texture and GBCD have been increasingly understood, as shown below.

\subsection{Effects of The Type and Sharpness of Texture}

It is well known that textured polycrystalline materials have special and anisotropic properties which are often of engineering importance (Bunge, 1984). As pointed out earlier, the properties of polycrystalline materials need to be understood in connection with grain boundaries and particularly GBCD. It is reasonable to consider that GBCD for a polycrystal with a special type of texture must be different from that for a randomly textured polycrystal from the view point of the localization of grain orientation distribution and a higher intensity of common rotation axis orientations for existing grain boundaries. It is interesting to study the GBCD in differently textured polycrystals of different materials in order to find some empirical law which relates the type and the sharpness of texture to GBCD. Howell et al. (1978) studied grain boundary structure in textured tungsten wires and rods by the transmission electron microscopy. They observed the presence of a high frequency of plane-matching type boundaries and coincidence boundaries, both of which are taken as low energy boundaries. It is feasible that a high frequency of low energy boundaries can occur in a strongly textured polycrystal in which neighbouring grains are more tightly correlated crystallographically and atomistically than in a random polycrystal. A breakthrough of quantitative understanding of the relationahip between texture and GBCD has been recently brought about by the work on the GBCD in differently textured iron- 6.5 mass $\%$ silicon ribbons produced by rapid solidfication and subsequent annealing (Watanabe et al., 1989a, 1989b). It was found that the type of texture can change drastically by annealing from random texture for as-solidified ribbon (or the ones slightly annealed at temperature lower than $1173 \mathrm{~K}$ or for short time at higher temperature) to $\{100\}$ or $\{110\}$-type texture for the ribbons fully annealed at $1363 \mathrm{~K}$ or $1473 \mathrm{~K}$ for $3.6 \mathrm{ks}$, respectively. The result of grain orientation distribution analysis for the differently textured ribbons is shown in Figure 4. The average grain size was $10 \mu \mathrm{m}$ for as-solidified ribbon and from $40 \mu \mathrm{m}$ to $800 \mu \mathrm{m}$ for the differently annealed specimens indicated. The charactaerization of grain boundaries in the annealed specimens was made and the distributions of grain boundary disorientation (one of the ways of the description of GBCD) are shown in Figure 5(a)-5(d). The disorientation distributions for the randomly textured ribbons (Figure 5(a) and 5(b)) are in good agreement with the curve predicted for a random polycrystal (Mackenzie, 1958). On the other hand the distributions for the $\{100\}$ or $\{110\}$-textured specimens greatly deviate from the curve, showing a characteristic distribution depending on the type of texture (Figure 5(c) and 5(d)). The presence of a high frequency of low angle boundaries (25\% for the $\{100\}$ and $17 \%$ for the $\{110\}$-texture) and of 

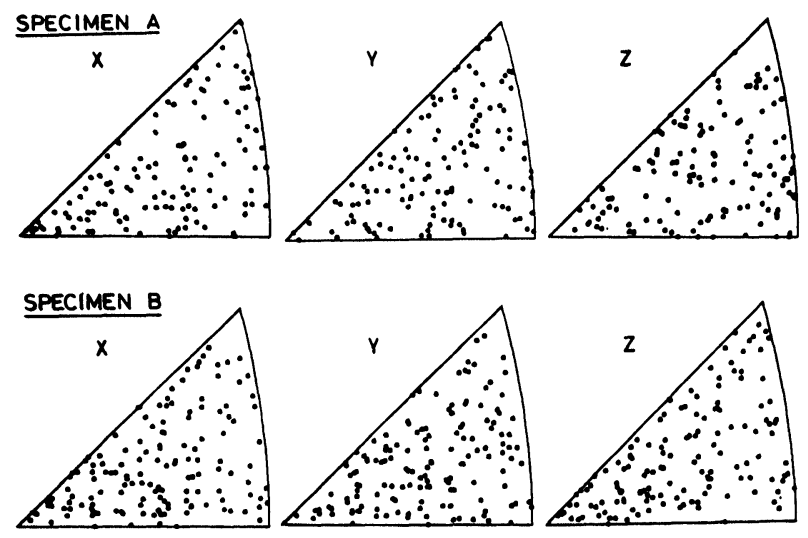

1100 ) TEXTURED RIBBON (Annealed at 1363K, for 3.6ks.)

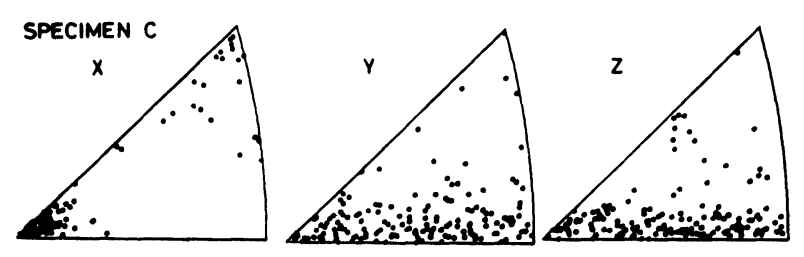

$1110)$ TEXTURED RIBBON (Annealed at $1473 \mathrm{~K}$ for $3.6 \mathrm{ks}$ )
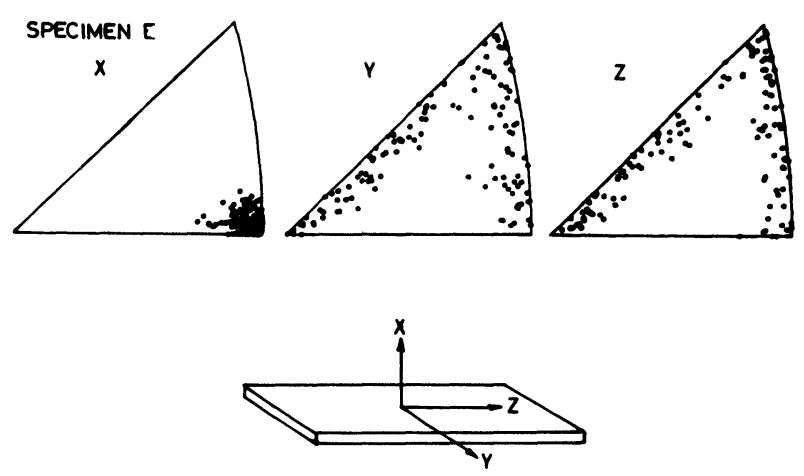

Figure 4 The distributions of grain orientations for Fe-6.5 mass \% Si ribbons annealed differently. Specimen A: $1173 \mathrm{~K}, 3.6 \mathrm{ks}$, Specimen B: $1363 \mathrm{~K}, 600 \mathrm{~s}$, Specimen C: $1363 \mathrm{~K}, 3.6 \mathrm{ks}$, Specimen D: $1473 \mathrm{~K}, 3.6 \mathrm{ks}$.

high angle boundaries with the disorientations around $55^{\circ}$ was observed. It was also found that low $\Sigma$ coincidence boundaries with specific $\Sigma$ values occurred preferentially with the frequencies in descending order of $\Sigma$ in the strongly textured ribbons but not for the randomly textured ribbons as shown in Figure 6(a) to 6(d). In the first order approximation, the $\Sigma$ values for those conicidence boundaries which occur preferentially in $\{\mathrm{hkl}\}$-textured polycrystal seem to be 




(a)

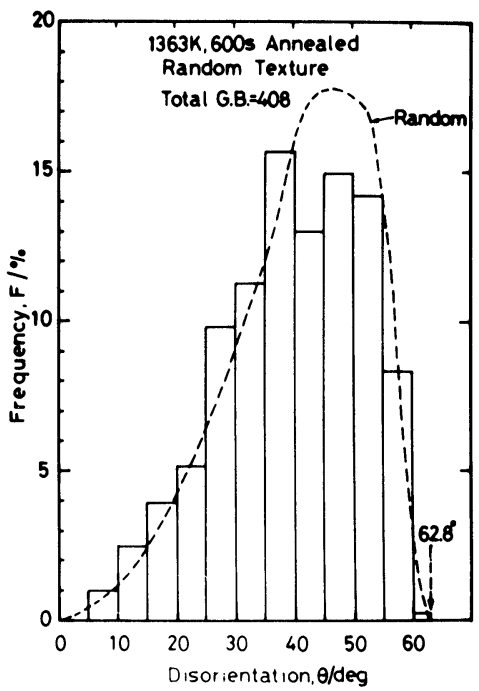

(b)

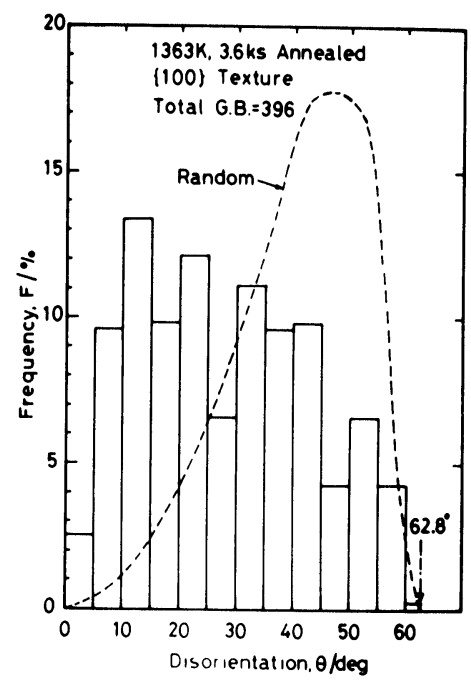

(c)

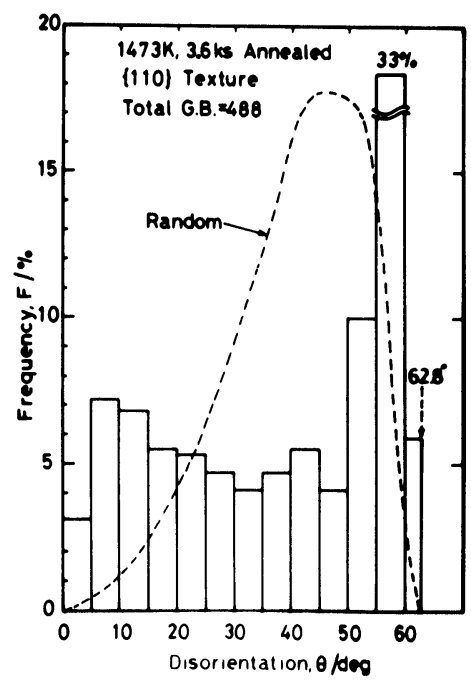

(d)

Figure 5 The distributions of grain boundary disorientations for $\mathrm{Fe}-6.5$ mass $\% \mathrm{Si}$ ribbons with different types of texture as same as indicated in Figure 4. 


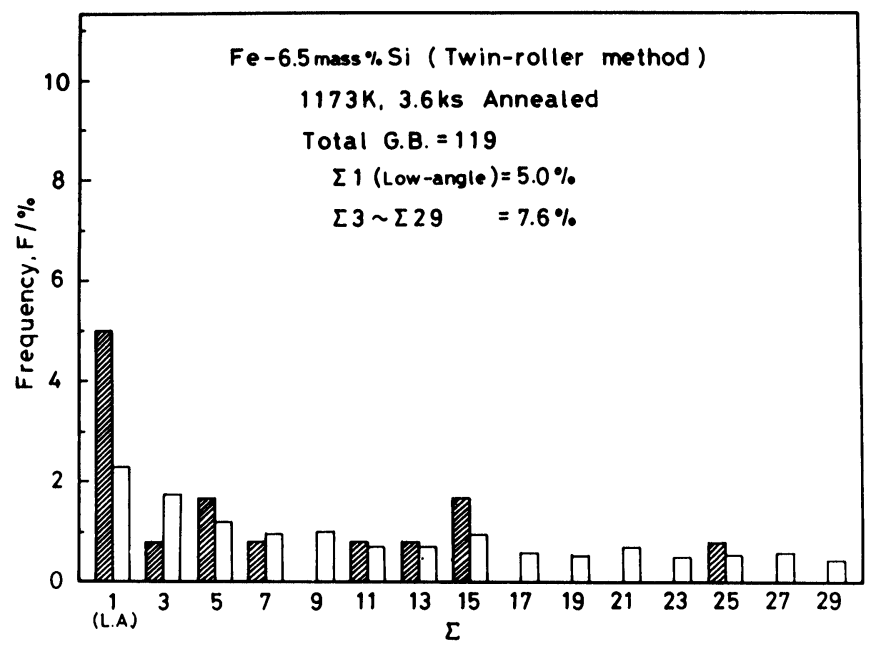

(a)

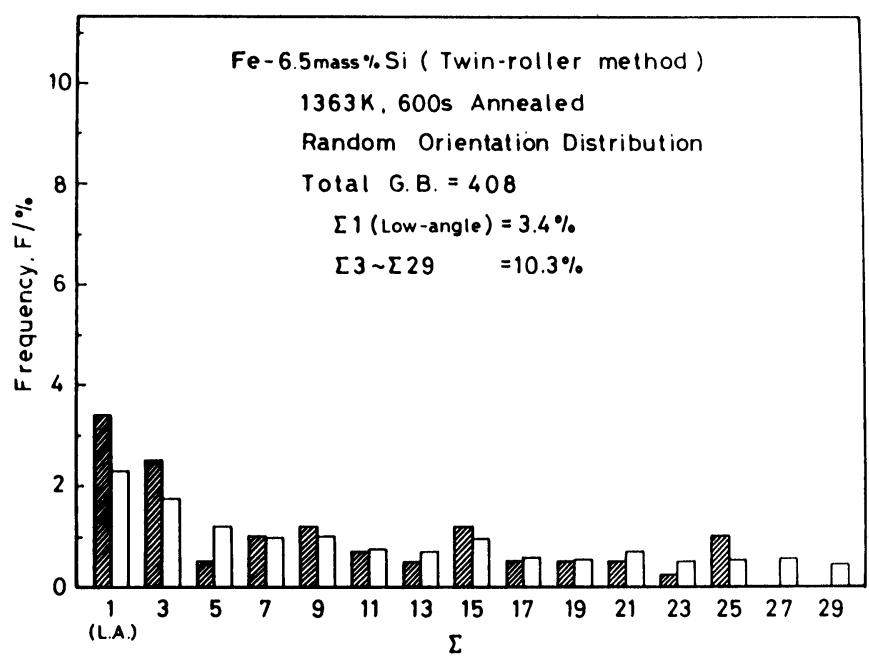

(b)

Figure 6 The frequency of coincidence boundaries as a function of $\Sigma$ for rapidly solidified and differently annealed Fe-6.5 mass \% Si ribbons. The speciments are the same as indicated in Figure 5.

predictable from possible coincidence orientations for the $\langle\mathrm{hkl}\rangle$ rotation. However it should be pointed out that for the $\{100\}$-textured iron-6.5\% silicon ribbon, $\Sigma 17$ coincidence boundary which is predicted for the $\langle 100\rangle$ rotation is missing and did not occur preferentially. This may suggest that the geometrical characterization of grain boundaries is useful and necessary for ordinary grain boundaries but not always sufficient for the characterization of special low energy boundaries which are determined by atomistic boundary parameters. The incorporation of a high frequency of low energy boundaries in the sharply 


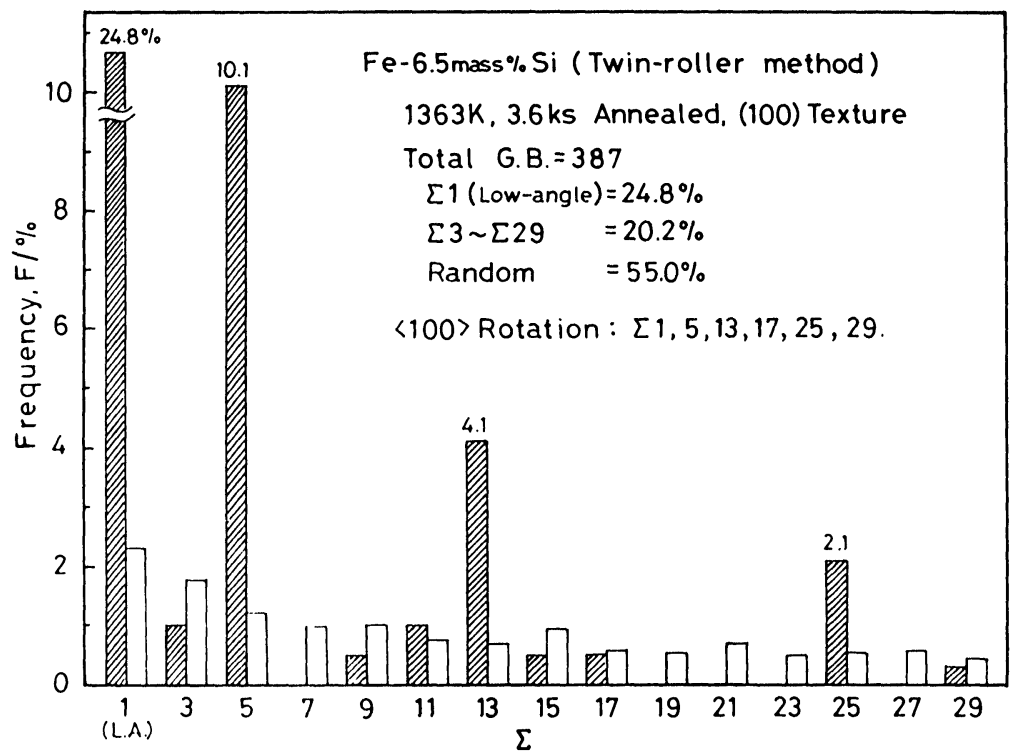

(c)

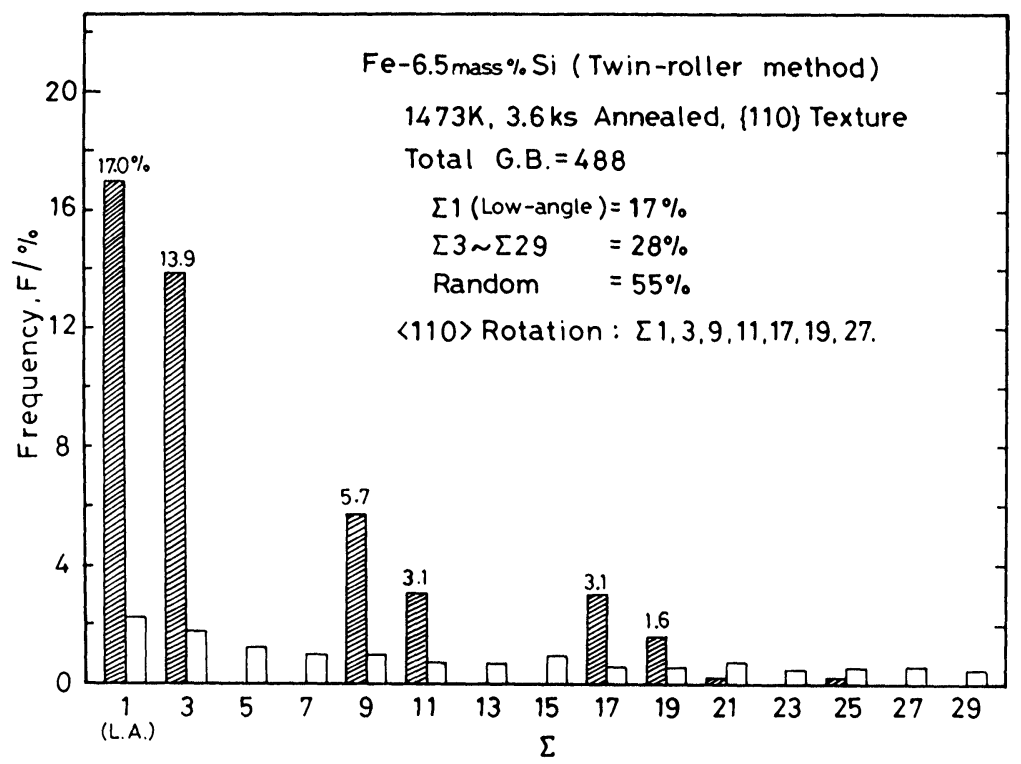

(d)

Figure 6 The frequency of coincidence boundaries as a function of $\Sigma$ for rapidly solidified and differently annealed $\mathrm{Fe}-6.5$ mass \% Si ribbons. The speciments are the same as indicated in Figure 5. 
textured iron-silicon ribbons into originally random-textured one has been attributed to the boundary migration and grain growth driven by the orientationdependent surface energy and the presence of a high frequency of random boundaries. Quite recently the frequency of coincidence boundaries in differently textured polycrystals has recently been calculated by Garbacz and Grabski (1989).

The most important recent finding associated with GBCD is probably the inverse cubic root $\Sigma$ dependence of the frequency of low $\Sigma$ coincidence boundaries as shown for the iron- 6.5 mass \% silicon ribbons in Figure 7 . It is evident that the frequency of $\Sigma$ coincidence boundaries, $F$ obeys the inverse cubic root $\Sigma$ dependence for $\Sigma 1, \Sigma 5, \Sigma 13$, and $\Sigma 25$ coincidence boundaries in the $\{100\}$-textured ribbon (Specimen C). Another interesting feature is that for the slightly annealed specimen $B$ which can be regarded as almost random polycrystal, the coincidence boundaries with $\Sigma 1, \Sigma 3 \Sigma 9, \Sigma 15$ and $\Sigma 25$ had the frequencies slightly exceeding the prediction for random polycrystal. The slope of the lines depends on the type of texture determined by annealing condition. Accordingly we can predict quantitatively the type and frequency of grain boundaries when the type of texture and the sharpness of texture are given in connection with annealing condition for a given material. A comparison between the two straight lines associated with the specimens $\mathrm{B}$ and $\mathrm{C}$, and the hatched band led us to realize some important aspects; the merging of these lines and the band at a certain $\Sigma$ value suggests that high incidence of coincidence boundaries caused by annealing may be possible only for the coincidence boundaries with $\Sigma$ smaller than the critical value around 29 . In other words these coincidence boundaries with $\Sigma$ smaller than the critical value may occur in an increasing frequency probably in connection with their boundary energies, as $\Sigma$ decreases up to 1 . Therefore the inverse cubic root $\Sigma$ dependence of the frequency of coincidence boundaries can be utilized as one of the criteria for low energy boundaries and for the upper bounds of $\Sigma$ value of physical significance. At present there is no decisive criterion for the upper bounds of $\Sigma$ applicable to real grain boundaries.

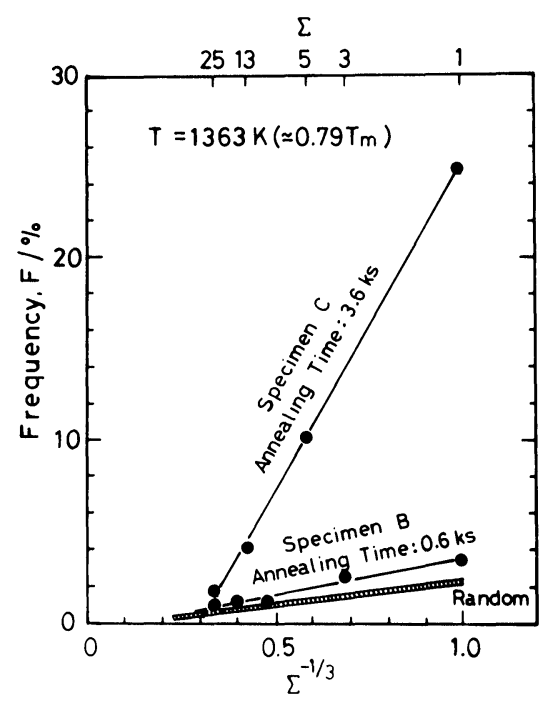

Figure 7 The inverse cubic root $\Sigma$ dependence of the frequency of coincidence boundaries for $\mathrm{Fe}-6.5$ mass \% $\mathrm{Si}$ ribbons with random texture (Specimen B) and with $\{100\}$ texture (Specimen C). 


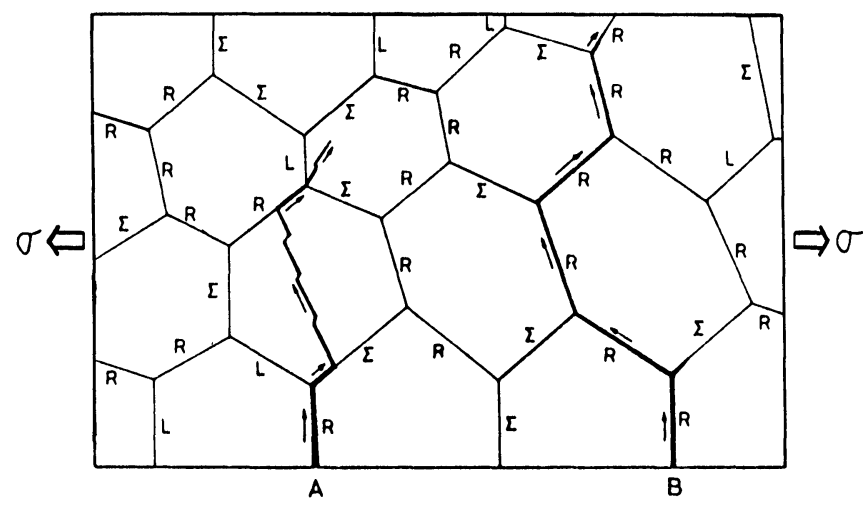

Figure 8 Schematic representation of grain boundary structure-dependent fracture processes in a polycrystal. Path A: combined process of intergranular and transgranular fracture. Path B: typical intergranular fracture.

\subsection{Other Microstructural Factors Associated with GBCD in Textured Polycrystal}

Figure 8 shows a schematic representation of grain boundary structure-dependent fracture processes occurring in a polycrystal (Watanabe, 1984), in which fracture mode and fracture characteristics are controlled primarily by GBCD (Lim and Watanabe, 1989, 1990) and by other GBCD-related microstructural factors discussed in this section. In a polycrystal the connection of random boundaries leads to crack propagation so that the local distribution and the configuration of high energy boundaries (reversely low energy boundaries) become important in addition to the averaged GBCD. We need to know the correlation between as more boundaries as possible interconnecting to each other with various geometrical configurations in the two- or three-dimensional polycrystal space.

4.2.1. Grain boundary triple lines (U-unit and I-unit). Grain boundaries of different characters meets other at triple lines or points with various geometrical configurations in a polycrystal. Recently Palumbo and Aust (1989) have pointed out the importance of the character of grain boundary triple lines to corrosion behaviour in nickel polycrystals. In real polycrystalline material the character of grain boundary triple lines is not always the same so that individual triple lines must be characterized depending on the type of grain boundaries meeting at a given boundary triple line, as originally proposed by Bollman (1984). Before the paper by Palumbo and Aust, much attention had not been paid to the characterization of boundary triple lines in connection with GBCD, although important roles of the triple lines in metallurgical phenomena were well recognized since very early (Smith, 1948) and quite recently several workers have carefully studied the effect of boundary triple lines on high temperature deformation and fracture (Miura et al., 1990). According to Bollmann there exist two types of boundary triple lines: U-unit and I-unit depending on whether the vector balance in obtained for grain boundary dislocations associated with three grain boundaries meeting at a triple line. The U-unit has a strain field due to a lack of vector balance so that this type of triple line is active in boundary-related 
phenomena, while the I-unit is less active. Such difference in the activity of triple lines is expected to produce some difference in boundary-related bulk properties of polycrystalline material Palumbo et al. (1991) have discussed a possibility of grain boundary design and control for intergranular stress corrosion resistance by controlling a fraction of different types of triple lines in connection with GBCD. It is reasonable to expect that the introduction of a high fraction of I-units can make a polycrystal more stress-corrosion resistant.

4.2.2. Grain boundary correlation number and length. As mentioned before, when high energy random boundaries are connected to each other, intergranular fracture can occur due to preferential crack propagation along random boundaries and results in a decrease in ductility of a polycrystal. Similarly it is suggested that the presence of a high fraction of random boundaries and their interconnection may enhance diffusion penetration in a polycrystal since random boundaries are known as highly efficient diffusional path (Swiatnicki et al., 1986). The geometrical configuration of like or unlike grain boundaries may be another important factor in addition to GBCD controlling the properties of polycrystalline material. Let us briefly discuss the importance of local geometrical configuration of different types of grain boundaries. In crack propagation not only GBCD but also the number of interconnecting random boundaries and their total length extending in a polycrystal are important (Watanabe, 1987). We may call these new microstructural factors associated with local GBCD "grain boundary correlation number and length". To the present author's knowledge such microstructural factors have never been discussed in order to explain and predict the properties of polycrystals until recently (Nichols et al., 1991). The introduction of a strong texture may be related to an increase of the correlation number of low energy boundaries while a random texture will bring about an increase of the correlation number of high energy boundaries. Simply speaking the correlation length can be related to GBCD and the average grain size, but when any local irregularity of the microstructure, for instance associated with abnormal grain growth, more detailed analysis of local GBCD is needed. Full understanding of boundary-related properties and behaviour of polycrystals must await future experimental and theoretical work on the boundary correlation number and length in relation to GBCD.

\section{GRAIN BOUNDARY DESIGN AND CONTROL FOR ADVANCED MATERIALS}

In recent years an increasing recognition of the importance of grain boundaries has been obtained among material scientists and engineers who are involved in development of advanced materials (Aust and Palumbo, 1989, Raj and Sass, 1988, Yoo, et al., 1988). As already pointed out by the present author (Watanabe, 1984, 1988a), grain boundaries have the great potential for future materials development. This section discusses recent achievement of the grain boundary design and control for advanced materials and some prospects for future work. 


\subsection{Toughening of Brittle Materials by the Control of Intergranular Fracture}

5.1.1. Prediction of toughening by modelling of fracture toughness. The brittleness associated with intergranular fracture has been a long pending problem in development of advanced structural materials. According to the idea of grain boundary design and control, the present author has already discussed the possibility of the control of the brittleness associated intergranular fracture by introducing a high frequency of low energy boundaries resistant to fracture (Watanabe, 1984, 1989). Recently Lim and Watanabe have made a modelling of fracture toughness and brittle-ductile transition as a function of GBCD for two-dimensional polycrystals (1989) and three-dimensional ones (1990). One of the important results obtained for three-dimensional polycrystal is shown in Figure 9. It was found that the fracture toughness of a polycrystal increases with increasing the fraction of low energy boundaries, $f$. As the ratio of the toughness for transgranular fraction $G_{T}$ and intergranular fracture $G_{I}$ increases, the enhancement of fracture toughness becomes more significant. This implies that the toughening by the introduction of low energy boundaries is the more effective for the material with the smaller boundary cohesion, that is more brittle material. Furthermore it was found that the brittle-ductile transition can occur when the fraction of low energy boundaries exceeds a certain critical value. Fracture mode changes from brittle intergranular fracture to ductile transgranular fracture so

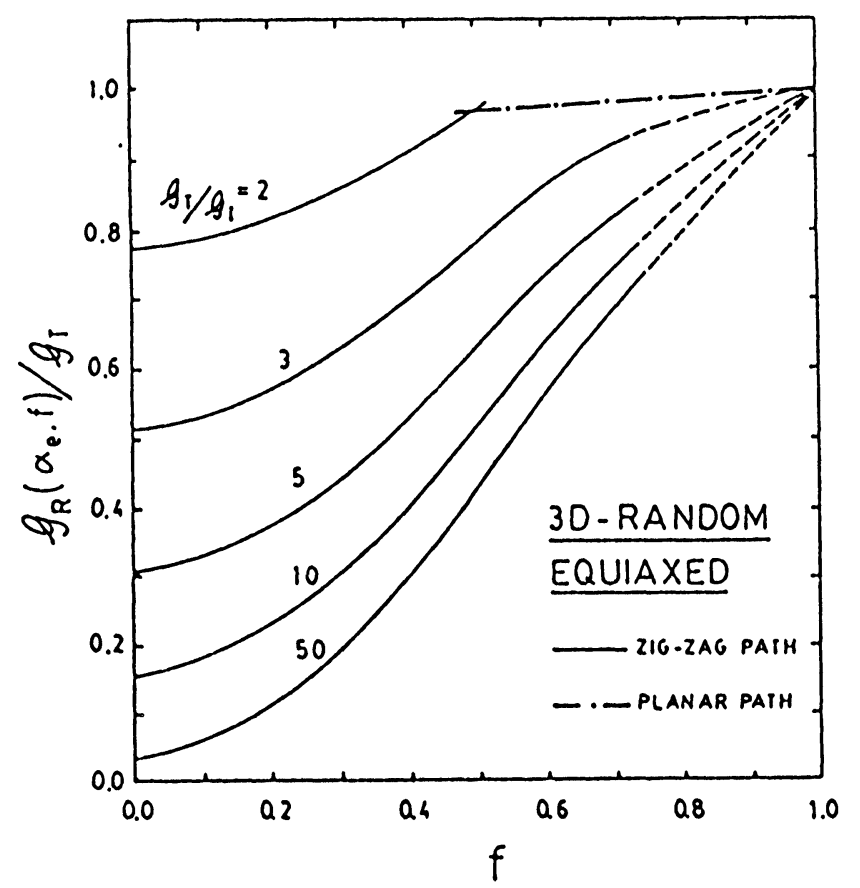

Figure 9 Effect of overall fraction of low-energy boundaries, $f$ and the ratio of the toughness associated with intergranular fracture and transgranular fracture, $G_{T} / G_{I}$ on the toughness of a three-dimensional polycrystal having a random GBCD. 
that brittle material becomes ductile. This kind of modelling can provide us useful information on the grain boundary design and control for intergranular fracture and brittleness through the design and control of GBCD. The present author has presented a guide line for the fraction of low energy boundaries $(1 / 3=33 \%)$ in order to toughen a polycrystal with a hexagonal configuration of grain boundaries (Watanabe, 1987). We can expect that a polycrystal which contains low energy boundaries by more than about $30 \%$ may exhibit reasonable ductility without incidence of intergranular fracture. Surprisingly this has been proved to work reasonably well as guide line, by experimental work on the GBCD in toughened polycrystals of "intrinsically brittle" materials.

5.1.2. Experimental evidence for toughening by grain boundary designcontrol. Iron-6.5mass\%silicon alloy is well known to be excellent magnetic material but difficult to be worked by conventional method. However Arai et al. (1986) have succeeded in ductilization of the material by rapid solidification and subsequent annealing. Lately the present author and coworkers (Watanabe et al., $1989 \mathrm{~b}$ ) have revealed that the origin of high ductility of rapidly solidified and annealed ribbon of the alloy can be ascribed to the presence of a high frequency $(45 \%)$ of low energy boundaries, well exceeding the guide line for toughening $(33 \%)$. This was an example of successful achievement of the control of brittleness by grain boundary control. The second case is concerned with a typical intermetallic material $\mathrm{Ni}_{3} \mathrm{Al}$ which is known to be brittle due to intergranular fracture. So far the addition of third element like boron has been only way to control the brittleness of this material. However recently Hirano $(1990,1991)$ has found that unidirectional solidification can drastically improve the brittleness of $\mathrm{Ni}_{3} \mathrm{Al}$ polycrystals; more than $50 \%$ elongation was obtained without addition of boron. Quite recently the present author and coworkers have observed the presence of a texture and a high frequency (more than 30\%) of low energy boundaries (mainly low-angle boundaries) in as-solidified $\mathrm{Ni}_{3} \mathrm{Al}$ polycrystals and as result of this the frequency of random boundaries was less than $60 \%$ (Watanabe et al., 1992). The frequency of low angle boundaries ranged from $21 \%$ to $57 \%$ depending on texture type. It is surprising to see that "intrinsically brittle" $\mathrm{Ni}_{3} \mathrm{Al}$ polycrystal can be ductilized by the introduction of more than about $40 \%$ low energy boundaries through unidirectional solidification by zone melting. This finding seems to have thrown a doubt about previous general recognition that the addition of boron is essential for ductilization of $\mathrm{Ni}_{3} \mathrm{Al}$ polycrystal. It should also be mentioned that quite recently "grain boundary pest" of boron-doped $\mathrm{Ni}_{3} \mathrm{Al}$ polycrystals at high temperature has been reported (Chuang et al., 1991). Since trace elements tend to segregate to grain boundary as temperature is raised, it is desirable to toughen $\mathrm{Ni}_{3} \mathrm{Al}$ polycrystals without any addition of third elements in order to use this material for high temperature service. The success of toughening $\mathrm{Ni}_{3} \mathrm{Al}$ polycrystal by the introduction of a high frequency of low-angle and low $\Sigma(3$ and 9$)$ coincidence boundaries (probably leading to an enhancement of slip transmission through grain boundaries and grain boundary cohesion) demonstrates the superiority of the grain boundary design and control though GBCD control as toughening method to other reported methods (Stoloff, 1989).

Let us take the third case of toughening of brittle material. Refractory metals like molybdenum and tungsten are known to be brittle due to the propensity to 
intergranular fracture. So grain boundaries in these metals have been often thought to be intrinsically brittle as in the case of intermetallics. However, recent systematic investigation of the effect of boundary type and structure on fracture has revealed that grain boundaries are not always brittle and low angle boundaries and low $\Sigma$ (at least 3 ) coincidence boundaries have much higher fracture strength than that of high angle random boundaries (Kurishita and Yoshinaga, 1989, Lim and Watanabe, 1990). It has been reported that molybdenum polycrystals produced thermomechanically from single crystal can be deformed even at room temperature but not for polycrystal which was initially polycrystalline (Brenner and Luft, 1982). This suggests that the GBCD can be changed depending on the detail of thermomechanical processing.

The present author and coworkers have recently studied the GBCD in molybdenum polycrystals produced by thermomechanical processing from single crystal sheets with different initial surface orientations (Watanabe et al., 1991). All the single crystal sheet specimens were deformed under simple plane strain condition by about $80 \%$ before annealing in vacuum. The thickness of the specimens was about $1 \mathrm{~mm}$. More than 300 boundaries were characterized for each specimen. It has been found that the frequency of low angle boundaries ranges from $20 \%$ to $35 \%$ depending on the initial orientation and annealing temperature. In two specimens (with the initial orientations of $\{111\}$ and $\{112\}$ ), low temperature annealing produced more low angle boundaries, but little change was observed for the specimen with the $\{110\}$ initial orientation. The frequency of low $\Sigma(3-29)$ coincidence boundaries ranged from $6 \%$ to $11 \%$. As result of this the frequency of random boundaries was from $55 \%$ to $72 \%$. The result of this GBCD analysis can explain the reason why molybdenum polycrystals produced from single crystal can be ductile. It may be safe to say that some but not all "intrinsically brittle" materials can be toughened and ductilized by introducing low energy boundaries with a higher frequency exceeding the critical value of about $30 \%$. It has already been discussed that metallic polycrystals produced thermomechanically contain as more low energy boundaries as the average grain size decreases. This well explains the general observation that polycrystalline materials become more ductile with decreasing grain size.

\subsection{Production of Desirable Properties by Introducing Desirable Boundaries}

Grain boundary design and control seem possible for functional materials with desirable properties and performance. This section discusses the possibility and prospect of the grain boundary design and development of different kinds of functional materials (corrosion-resistant metallic material, high performance superconducting oxide and ferromagnetic materials) through the control of GBCD.

5.2.1. Corrosion-resistant materials. The effect of boundary type and misorientation on boundary corrosion was established experimentally by using bicrystal specimens whose boundary character was systematically controlled (Froment, 1975, Yamashita et al., 1991). Low angle boundaries and low $\Sigma$ coincidence boundaries are resistant to corrosion but high angle random boundaries are prone to preferential corrosion. On the basis of observations of structure-dependent boundary corrosion, we can expect that a polycrystal with a high fraction of low 

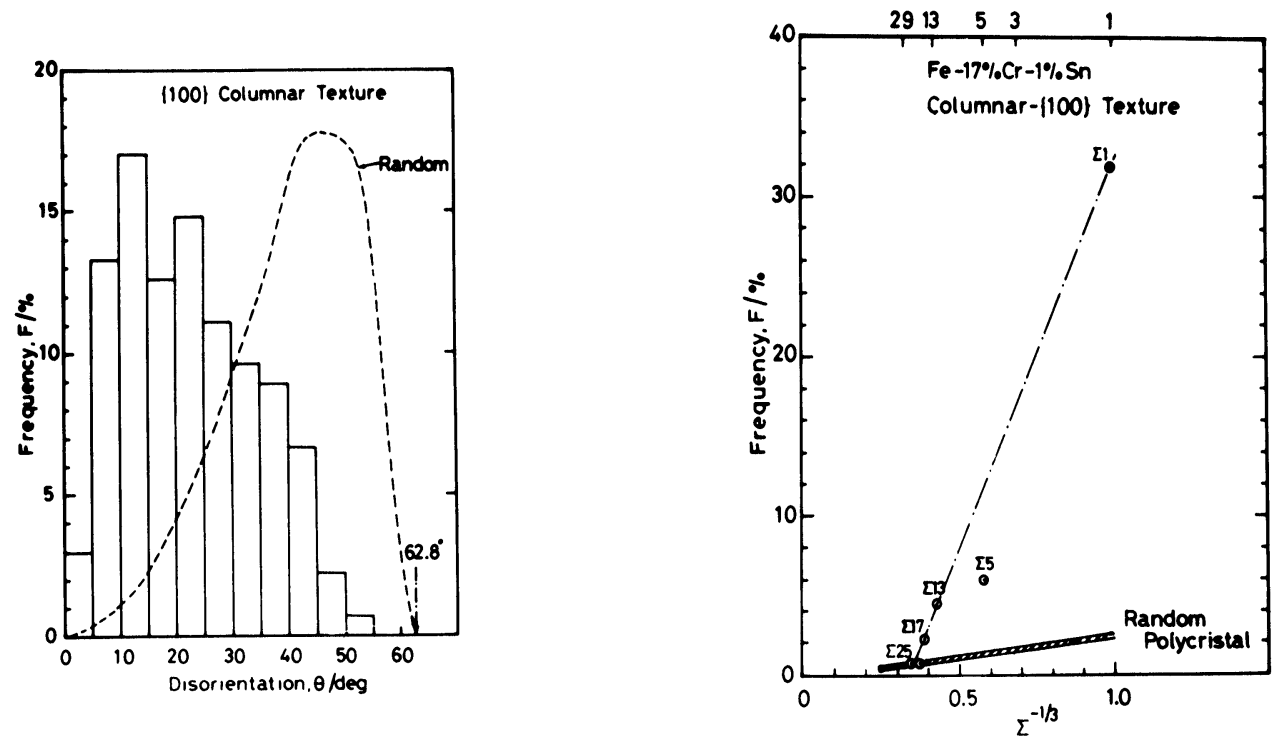

Figure 10 (a) The distribution of grain boundary disorientations and (b) the inverse cubic root $\Sigma$ dependence of the frequency of coincidence boundaries for cast Fe-16 mass \% Cr-1 \% Sn alloy polycrystal with $\{100\}$ columnar-texture.

energy boundaries would be more corrosion-resistant than a polycrystal with a smaller fraction of low energy boundaries even for the material with the same chemical composition. The present author and coworkers (Watanabe et al., 1988) have observed that as solidified iron-17 mass \% chromium alloy which had columnar grain structure and $\langle 100\rangle$ texture, contains high frequencies of low angle boundaries and low $\Sigma(\Sigma 5, \Sigma 13, \Sigma 17)$ coincidence boundaries as shown in Figure 10(a) and 10(b). It is evident that the level of the frequency of the low energy boundaries is much higher than that predicted for a random polycrystal. The inverse cubic root $\Sigma$ dependence almost holds for the frequencies of these low energy boundaries in the $\langle 100\rangle$-textured iron-chromium alloy sample which shows higher corrosion resistance than ordinarily produced sample without any texture. Hu and Goodman (1984) previouly discussed the possibility of improvement of corrosion and creep resistance of stainless steel by introducing a strong cube texture through recrystallization of rolled strip. As has been discussed so far, the physical meaning of the introduction of a texture may be understood from GBCD point of view. Palumbo et al. (1991) recently confirmed the possibility of development of stress corrosion-resistant materials by grain boundary design and control.

5.2.2. Electronic superconducting ceramics. The distinctive role of individual grain boundaries in structure-dependent electrical properties of polycrystalline electronic and superconducting ceramics has been increasingly recognized (Clarke, 1990). Systematic investigation of the effect of boundary type and structure on current/voltage characteristics in electronic ceramics has just recently 
started to be studied (Olsson and Dunlop, 1989), although essential role of grain boundary in varistor material like $\mathrm{ZnO}$ was already known. It has been confirmed that the current/voltage characteristics strongly depends on the type and structure of grain boundary. Similarly in the case of superconducting material like $\mathrm{YBa}_{2} \mathrm{Cu}_{3} \mathrm{O}_{7-x}$. it was found that the ratio of the critical current density of tilt boundary to the average value of the grain interior decreases systematically with increasing tilt angle by almost two orders of magnitude over the range from $0^{\circ}$ to $35^{\circ}$ reaching a saturation value of about $1 / 50$ at a tilt angle of $20^{\circ}$ (Dimos et al., 1988). However until recently there has been no information on GBCD data for this material so that the important data on misorientation dependence of the critical current density has not been correlated to the average value of bulk polycrystalline sample. Quite recently Zhu et al. (1991) have studied the GBCD in $\langle 100\rangle$-textured $\mathrm{YBa}_{2} \mathrm{Cu}_{3} \mathrm{O}_{7-x}$ and observed the presence of a high frequency of low angle boundaries (more than $70 \%$ of observed boundaries). They consider that the observed improvement of superconducting characteristics of their sample is ascribed to the presence of a high fraction of low energy boundaries. This work is a good example which can demonstrate the importance and usefulness of GBCD in understanding and controlling grain boundary-related properties in functional polycrystalline materials. However it is necessary to study the relationship between GBCD and processing method and condition in order to design and to precisely control desirable boundaries. Unfortunately this kind of information is extremely scarce at present. Much work remains open to be done.

5.2.3. Ferromagnetic materials. The role of grain boundary in ferromagnetic materials has not been well understood particularly in connection with boundary type and structure. It was reported before that when deformed alpha iron was annealed in a magnetic field, a texture was generated probably due to the magnetic anisotropy (Martikainen and Lindroos, 1981). The present author and coworkers have studied the effect of magnetic annealing on recrystallization and GBCD in iron-9 mol \% cobalt alloy (Watanabe et al., 1990). It was found that the magnetic annealing retards recrystallization and affects GBCD, particularly the frequency of low angle boundaries in recrystallized polycrystals of the alloy. As shown in Figure 11, the frequency of low angle boundaries increases almost linearly up to about $8 \%$ of observed boundaries being 3.4 times larger than the prediction for random polycrystal with increasing magnetic field strength up to $5 \mathrm{k} 0 \mathrm{e}$. The application of much higher magnetic field strength is expected to produce a stronger texture and a higher frequency of low angle boundaries than the observation in this work. The magnetic annealing may be applicable to other magnetic materials in order to control the GBCD through texture evolution and we can expect a stronger effect if the material has a larger magnetic anisotropy. Since the magnetic annealing was found to retard recrystallization, it is interesting to study whether this method can produce a texture and desirable GBCD without abnormal grain growth which is often involved in texture evolution and produces detrimental effect on bulk properties of polycrystalline materials.

\section{CONCLUSION}

The present paper has discussed the possibility of grain boundary design and control for advanced structural and functional polycrystalline materials, on the 


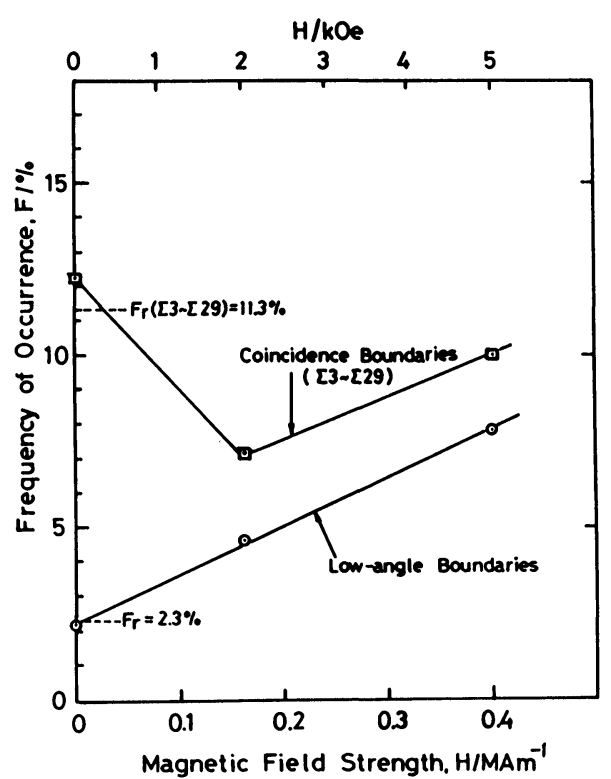

Figure 11 The effect of magnetic annealing on the frequency of low-angle boundaries and coincidence boundaries in $\mathrm{Fe}-9 \mathrm{~mol} \% \mathrm{Co}$ alloy polycrystal.

basis of recent finding of the close relationship between texture and grain boundary character distribution (GBCD). The relationship between texture and GBCD has been discussed from several aspects. Other microstructural factors associated with GBCD has been introduced and discussed. It has been demonstrated that GBCD can be used as a powerful tool for designing and controlling grain boundaries in order to endow polycrystalline materials with desirable properties and performance. Several cases have been introduced where grain boundary design and control were successfully achieved for the development of advanced materials, particularly referring to recent work on toughening of brittle materials by controlling GBCD. The possibility of grain boundary design and control for several advanced functional materials has been discussed.

\section{Acknowledgements}

The author wishes to thank coworkers for their contributions to the work referred in this paper. He also wishes to thank Professor C. Esling of the University of Metz, France, for critical reading the manuscript and the provision of a pleasant stay as an Invited Professor, during which this paper was prepared and presented. This work was supported by a Grant-in-Aid for Research from the Ministry of Education, Science and Culture, Japan.

\section{References}

Abbruzzese, G. and Lücke, K. (1986). Acta Metall., 34, 905-914.

Arai, K.-I. and Ohmori, K. (1986). Metall. Trans., 17A, 1295-1299.

Aust, K. T. (1981). Prog. Mater. Sci., Chalmers Anniversary Volume, 27-48. 
Aust, K. T. and Palumbo, G. (1989). Proc. Intern. Symp. on Advanced Structural Materials, ed. by Wilkinsons, D. S., Pergamon Press, 215-226.

Balluffi, R. W. (ed) (1980). Grain Boundary Structure and Kinetics, ASM.

Belluz, R. V. and Aust, K. T. (1975). Metall. Trans., 6A, 219-220.

Biscondi, M. (1984). Physical chemistry of the solid state, Applications to metals and their compounds, ed. by P. Lamcombe, Elsevier Sci. Pub. 225-241.

Bollmann, W. (1984). Phil. Mag., A49, 73-79.

Bunge, H. J. (1984). Proc. 7th Intern. Conf. on Textures of Materials, Netherlands Soc. Mater. Sci., 447-464.

Brenner, B. and Luft, A. (1982). Mater Sci. Eng., 229-237.

Chung, T. H., Pan, Y. C. and Hsu, S. E. (1991). Metall. Trans., 22A, 1801-1809.

Clarke, D. R., Proc. Intern. Cong. on Intergranular and Interphase Boundaries, Colloq. de Phys., suppl., 51, C1-935-943.

Dechamps, M. (1991). Textures and Microstructures, 14-18, 733-738.

Dimos, D., Chaudhari, P., Mannhart, J. and LeGues, F. K. (1988) Phys. Rev. Lett. 61, 219-222.

Froment, M. (1975). J. de Phys., 36, C4-371-385.

Fukutomi, H. and Horiuchi, R. (1981). Trans. Japan Inst. Met., 22, 633-642.

Garbacz, A. and Garbski, M. W. (1989). Scripta Metall., 23, 1369-1374.

Gleiter, H. and Chalmers, B. (1972). Prog. Mater. Sci., Pergamon Press, 16, 1-272.

Harase, J., Shimizu, R. and Watanabe, T. (1988). Proc. 8th Intern. Conf. on Textures of Materials (ICOTOM-8), TMS., 723-728.

Hirano, T. (1990). Acta Metall. Mater., 38, 2667-2671.

Hirano, T. (1991). Scripta Metall. Mater., 25, 1747-1750.

Honeycombe, R. W. K. (1983). Trans. Japan Inst. Metals, 24, 177-189.

Howell, P. R., Fleet, D. E., Welch, P. I. and Ralph, B. (1978). Acta Metall., 26, 1499-1503.

$\mathrm{Hu}, \mathrm{H}$. and Goodman, S. R. (1984). Mater. Sci. Eng., 67, 143-155.

King, A. H. (1987). Intern. Mater. Reviews, 32, 173-189.

Kurishita, H. and Yoshinaga, H. (1989). Materials Forum, 13, 161-173.

Lagarde, P. and Biscondi, M. (1974). Canadian Metall. Quart., 13, 245-251.

Lim, L. C. and Raj, R. (1984). Acta Metall., 32, 1177-1181.

Lim, L. C. and Watanabe, T. (1989). Scripta Metall., 23, 489-494.

Lim, L. C. and Watanabe, T. (1990). Acta Metall. Mater., 38, 2507-2516.

Mackenzie, J. K. (1958) Biometrika, 45, 229-240.

Martikainen, H. O. and Lindroos, V. K. (1981). Scan. J. Metall., 10, 3-8.

Miura, S., Okada, T., Onaka, S. and Hashimoto, S. (1990). Proc. Intern. Cong. on Intergranular and Interphase Boundaries in Metals, Colloq. de Phys., 51, C1-587-592.

Nichols, C. S., Cook, R. F., Clarke, D. R. and Smith, D. A., (1991) Acta Metall. Mater., 39, 1657-1665.

Olsson, E. and Dunlop, G. L. (1989). J. Appl. Phys., 66, 3666-3675.

Otsuki, A. and Mizuno, M., (1986). Proc. JIM Symp. on Grain Boundary Structure and Related Phenomena, Trans. Japan Inst. Metals, 27, suppl., 789-796.

Palumbo, G. and Aust, K. T. (1989). Proc. Intern. Symp. on Advanced Structural Materials, ed. by Wilkinsons, D. S., Pergamon Press, 227-233.

Palumbo, G., King, P. J., Aust, K. T., Erb, U. and Lichtenberger, P. C. (1991). Scripta Metall. Mater., 25, 1775-1780.

Chadwick, G. A. and Smith, D. A. (ed). (1976). Grain Boundary Structure and Properties, Academic Press, London.

Raman, V., Watanabe, T. and Langdon, T. G. (1989). Acta Metall., 37, 705-714.

Randle, V. and Brown, A. (1989). Phil. Mag., A59, 1075-1089.

Randle, V., Ralph, B. and Dingley, D. (1988). Acta Metall., 36, 267-273.

Raj, R. and Sass, S. L. (ed.) (1988). Proc. Intern. Conf. on Interface Science and Engineering, 87, J. de Phys., 49, C5.

Rühle, M., Balluffi, R., Fishmeister, H. and Sass, S. L. (ed.) (1985). Proc. Intern. Conf. on The Structure and Properties of Internal Interfaces, J. de Phys., 46, C4.

Schmelzle, R., Muschik, T., Gust, W. and Predel, B. (1991). Scripta Met. Mater., 25, 1981-1986.

Shimizu, R. and Harase, J. (1989). Acta Metall., 37, 1241-1249.

Smith, C. S. (1948). Trans. AIME., 175, 15-51.

Stoloff, N. S. (1989). Intern. Mater. Reviews, 34, 153-183.

Swiatnicki, W. A., Lojkowski, W. and Grabsski, M. W. (1986). Acta Metall., 34, 599-605.

Watanabe, T. (1983a). Phil Mag., 47, 141-146. 
Watanabe, T. (1983b). Metall. Trans., 14A, 531-545.

Watanabe, T. (1984). Res Mechanica, 11, 47-84.

Watanabe, T. (1986). Proc. 4th JIM Intern. Symp. on Grain Boundary Structure and Related Phenomena, Trans. Japan Inst. Metals, 27, suppl., 73-82.

Watanabe, T. (1987). Chemistry and Physics of Fracture, ed. by Latanision, R. M. and Jones, R. H., NATO ASI Series No 130, Martinus Nijhoff Pub., 492-497.

Watanabe, T. (1988a). MRS Symp. on Interfacial Structure, Properties and Design, ed. by Yoo, M. H., Clark, W. A. T. and Briant, C. L., MRS, 122, 443-454.

Watanabe, T. (1988b). Materials Forum, 11, 284-303.

Watanabe, T. (1989). Materials Science Forum, 46, 25-48.

Watanabe, T. (1990). Proc. Intern. Conf. on Recrystallization in Metallic Materials, ed. by T. Chandra, TMS., 405-410.

Watanabe, T., (1992). Proc. Intern. Conf. on Grain Growth in Polycrystalline Materials, Materials Science Forum, 94-96, 209-220.

Watanabe, T., Arai, K.-I., Yoshimi, K. and Oikawa, H. (1989a). Phil Mag. Letters, 59, 47-52.

Watanabe, T., Fujii, H., Oikawa, H. and Arai, K.-I. (1989b). Acta Metall., 37, 941-952.

Watanabe, T., Kitamura, S. and Karashima, S. (1980). Acta Metall., 28, 455-463.

Watanabe, T., Suzuki, Y., Tanii, S. and Oikawa, H. (1990). Phil. Mag. Letters, 62, 9-17.

Watanabe, T., Takazawa, M. and Oikawa, H., (1988). Proc. 8th Intern. Conf. on the Strength of Metals and Alloys (ICSMA-8), Pergamon Press, 1357-1362.

Watanabe, T., Hirano, T., Ochiai, T. and Oikawa, H. (1992). Unpublished.

Watanabe, T., Kobylanski, A., Kishino, J., Ando, T. and Oikawa, H. (1991). Unpublished.

Wyrzykowski, J. W. and Grabski, M. W. (1986). Phil. Mag, A53, 505-520.

Yamashita, M., Hashimoto, S., Mimaki, T. and Miura, S. (1991). Mater. Trans., 32, 885-897.

Yoo, M. H., Clark, W. A. T. and Briant, C. L. (ed) (1988). Interfacial Structure, Properties and Design, Materials Research Society (MRS), Vol. 122.

Zhu, Y., Zhang, H., Wang, H. and Seunaga, M. (1991). J. Mater. Res., 6, 2507-2518. 